\title{
RESPOND - a patient-centred programme to prevent secondary falls in older people presenting to the emergency department with a fall: protocol for a multicentre randomised controlled trial
}

\author{
A L Barker, ${ }^{1}$ P A Cameron, ${ }^{1}$ K D Hill, ${ }^{2}$ L Flicker, ${ }^{3,4,5}$ T P Haines, ${ }^{6}$ J A Lowthian, ${ }^{1}$ \\ N Waldron, ${ }^{7} \mathrm{G}$ Arendts, ${ }^{3,5}$ J Redfern, ${ }^{8}$ A Forbes, ${ }^{9}$ C A Brand, ${ }^{1,10}$ \\ C D Etherton-Beer, ${ }^{3,4,5}$ A M Hill, ${ }^{11}$ P Hunter, ${ }^{12}$ S R Nyman, ${ }^{13}{ }^{13}$ Smit ${ }^{12}$
}

For numbered affiliations see end of article.

\section{Correspondence to} Dr Anna Barker, DEPM, The Alfred Centre, 99 Commercial Road, Melbourne, VIC 3004, Australia; anna.barker@monash.edu

Received 14 April 2014 Revised 23 April 2014 Accepted 29 April 2014 Published Online First 23 June 2014

CrossMark

To cite: Barker $A L$, Cameron PA, Hill KD, et al. Inj Prev 2015;21:e1.

\begin{abstract}
Introduction Participation in falls prevention activities by older people following presentation to the emergency department (ED) with a fall is suboptimal. This randomised controlled trial (RCT) will test the RESPOND programme, an intervention designed to improve older persons' participation in falls prevention activities through delivery of patient-centred education and behaviour change strategies.
\end{abstract}

Design and setting $A R C T$ at two tertiary referral EDs in Melbourne and Perth, Australia.

Participants 528 community-dwelling people aged 60-90 years presenting to the ED with a fall and discharged home will be recruited. People who require an interpreter or hands-on assistance to walk; live in residential aged care or $>50 \mathrm{~km}$ from the trial hospital; have terminal illness, cognitive impairment, documented aggressive behaviour or a history of psychosis; are receiving palliative care or are unable to use a telephone will be excluded.

Methods Participants will be randomly allocated to the RESPOND intervention or standard care control group. RESPOND incorporates (1) a home-based risk factor assessment; (2) education, coaching, goal setting and follow-up telephone support for management of one or more of four risk factors with evidence of effective interventions and (3) healthcare provider communication and community linkage delivered over 6 months. Primary outcomes are falls and fall injuries per person-year. Discussion RESPOND builds on prior falls prevention learnings and aims to help individuals make guided decisions about how they will manage their falls risk. Patient-centred models have been successfully trialled in chronic and cardiovascular disease; however, evidence to support this approach in falls prevention is limited.

Trial registration number The protocol for this study is registered with the Australian New Zealand Clinical Trials Registry (ACTRN12614000336684).

\section{BACKGROUND}

Falls are one of the leading causes for emergency department (ED) presentations in older people. ${ }^{1}$ In the 6 months following an index fall ED presentation, up to $52 \%$ of cases experience subsequent falls, ${ }^{2}{ }^{3} 49 \%$ are re-hospitalised and many experience functional decline. ${ }^{2}$

There is conflicting evidence surrounding the effect of programmes designed to reduce secondary falls in older people presenting to the ED with a fall. Eight studies have reported programmes that had no effect on new falls, fall injuries or ED presentations, ${ }^{4-11}$ while three reported programmes reduced secondary falls. ${ }^{3} 1213$ The characteristics that appear to differentiate successful programmes from others include delivery of the intervention within 1 month of the index fall and greater intensity of the interventions. ${ }^{14}$ An Australian randomised controlled trial (RCT) of older people attending the ED after a fall reported that for patients who accessed falls prevention services recommended by project staff after baseline assessment (an average of 28 days after ED presentation), the time lag to service access was too long4 months for falls clinics, 2 months for physiotherapy and 3 months for occupational therapy. ${ }^{4}$ Similar delays were reported in a Dutch RCT that used an interdisciplinary intervention ${ }^{6}$ and a Danish RCT, where the time lag from fall to intervention was 7 weeks. ${ }^{15}$ In contrast, a successful UK trial delivered services within 1 month of ED discharge. ${ }^{12}$

Poor patient participation in falls prevention activities also appears to be an important factor underpinning the effectiveness of prior programmes and may be related to care not centring on what the patient perceives as being important. ${ }^{16} 17$ The Australian RCT cited $<5 \%$ of people presenting the ED with a fall subsequently attend falls clinics, $<30 \%$ attend physiotherapy and $<17 \%$ attend occupational therapy. ${ }^{4}$ These findings of limited patient participation in prevention activities are consistent with an Australian qualitative study that reported that $72 \%$ of patients (with a fall-related ED presentation) were reluctant to attend exercise classes, 59\% were hesitant to cease psychotropic medications and 43\% were unwilling to have a home safety assessment. ${ }^{16}$ Conversely, older people see relevance in falls prevention strategies that adopt a patient-centred approach by including education and involvement in decision making. ${ }^{18}$ Guidelines to increase uptake of falls prevention strategies have also suggested older adults choose activities that have personal meaning and are compatible with their social norms. ${ }^{19}$

Patient-centred care models have been successfully trialled in chronic disease and secondary prevention of cardiovascular events. ${ }^{20}$ A RCT of 144 patients 
with acute coronary syndrome tested the 'Choice of Health Options In prevention of Cardiovascular Events (CHOICE)' programme. CHOICE showed that a brief patient-centred programme comprising a clinic visit and telephone support resulted in significant improvement in cardiac risk profiles compared with profiles of patients receiving standard care. ${ }^{21}$ Importantly, a follow-up study found CHOICE participants maintained favourable changes in coronary risk profile at 4 years compared with controls, indicating that a brief patient-centred programme with telephone support is an effective long-term intervention. ${ }^{22}$

Incorporating patient-centred care principles and telephone support into falls prevention programmes may improve participation in falls prevention strategies. This approach is supported by a recent review that reported participation in falls prevention strategies was highest in studies that offered moderate home visit support and intervention via telephone contact, where moderate support was defined as less than one home visit or telephone call per month and more than two home visits in total. $^{23}$ Presenting information as positive health messages or as 'life enhancing' rather than 'at risk' may also improve participation. ${ }^{19}$

The efficacy of patient-centred falls prevention programmes that include education and coaching via positive health messages to address falls risk factors has not been previously reported. The current study will address this evidence gap by investigating the impact of a patient-centred falls prevention programmeRESPOND—on the rate of falls, fall injuries and ED re-presentations in older people presenting to the ED with a fall. The objectives of this paper are to describe the protocol for this trial.

\section{METHODS}

\section{Design}

A single-blind multicentre RCT of the RESPOND programme compared with falls risk assessment and standard postdischarge care will be conducted. Figure 1 outlines each step of the study.

\section{Participants and setting}

Community-dwelling persons aged 60-90 years who present over a 12 -month period to two large, metropolitan, tertiary referral major trauma centre EDs with a fall and who are planned to be discharged directly home from the hospital within $72 \mathrm{~h}$ will be recruited during their hospital stay. This study targets patients who are planned to have a short inpatient stay as these people are least likely to receive comprehensive geriatric assessment and management and would therefore be at greater risk of secondary falls than patients hospitalised for longer periods or discharged to rehabilitation services.

Exclusion criteria relate to an inability to participate in the intervention and include discharge to residential aged care, current palliative care or terminal illness, requiring hands-on assistance to walk, being unable to use a telephone, needing an interpreter and presence of cognitive impairment, social aggression or a history of psychoses. As a reflection of study constraints around home visits, people living further than $50 \mathrm{~km}$ from the trial hospital will also be ineligible to participate.

\section{Sample size}

The study is powered to detect a significant difference in the primary outcome of the rate of falls and fall injuries between the intervention and control groups in the 12-month follow-up. Assuming a control group fall injury rate of 1.01 injuries per person-year, ${ }^{4}$ we require 293 participants to have $80 \%$ power to detect a rate ratio of 0.70 between intervention and control groups at the $5 \%(z=2.8)$ significance level. To allow for a $20 \%$ loss to follow-up ${ }^{4}$ and over-dispersion $(\phi=1.5) 528$ participants $(n=264$ per group) are required. The study will be adequately powered to detect differences in ED re-presentations in the 12-month follow-up based on an expected control rate of 0.71 per person-year, ${ }^{4}$ and $80 \%$ power to detect a rate ratio of 0.70 between intervention and control groups at the $5 \%$ significance level $(\mathrm{N}$ required $=502)$.

\section{Recruitment}

A three-stage process will be used by research staff to identify eligible participants. Stage 1 involves screening electronic records on a daily basis in the ED to identify potential participants based on age, living status (home as opposed to residential aged care), presenting diagnosis and distance of home from the hospital. Stage 2 involves review of medical records of persons meeting stage 1 screening requirements to determine those who meet the inclusion criteria of planned discharge home within $72 \mathrm{~h}$ and to exclude people who have a documented life expectancy of 12 months or less, are receiving palliative care or have a history of social aggression or psychoses. Stage 3 involves approaching people meeting stage 2 screening requirements to obtain verbal consent to conduct a screening interview. During the interaction, the research staff will determine whether the individual requires an interpreter, is able to use the telephone, has a hearing impairment or requires physical assistance from another person to walk. Cognitive ability will be determined by the Mini Mental State Examination (MMSE) ${ }^{24}$ applying a cut-off score of $<23$. Potential participants who have a physical impairment or injury that limits upper limb function will have the MMSE score adjusted as per the tool's handbook. ${ }^{25}$

Eligible participants at this stage will be provided with an overview of the study including written information about the study and asked to provide written consent to participate.

\section{Randomisation}

After receipt of informed written consent, participants will be randomly assigned into one of the two trial groups. A web-based randomisation sequence will be used, with permuted block randomisation stratified by recruitment site to ensure equal control and intervention participant numbers across sites. Research staff will be unaware of the next group allocation at the time that they request a participant's group assignment. The participants and research staff will be blinded to group allocation until after the baseline assessment has been completed.

\section{Baseline assessment}

The next phase of the study is conducted by the RESPOND clinician-a registered healthcare professional, who will visit the participant at their home within 2 weeks of discharge from hospital. At this visit, data will be collected relating to demographic details, social history, index and past fall history, existing referrals and any clinical recommendations made by hospital staff. A falls risk factor assessment will be completed and falls selfefficacy, functional health literacy and health-related quality of life will also be evaluated (figure 2).

The Falls Risk for Older People in the Community (FROPCom) assessment will be used to assess falls risk factors and risk status (low, medium, or high falls risk). This tool includes 28 questions on 13 risk factors that have either dichotomous or ordinal scoring from 0 to 3 . A total score out of 60 is obtained with higher scores indicative of greater risk. ${ }^{56}$ High inter-rater and intra-rater reliability has been reported as has a moderate accuracy to predict those at risk of future falls. ${ }^{5} 26$ 
Figure 1 Participant flow.

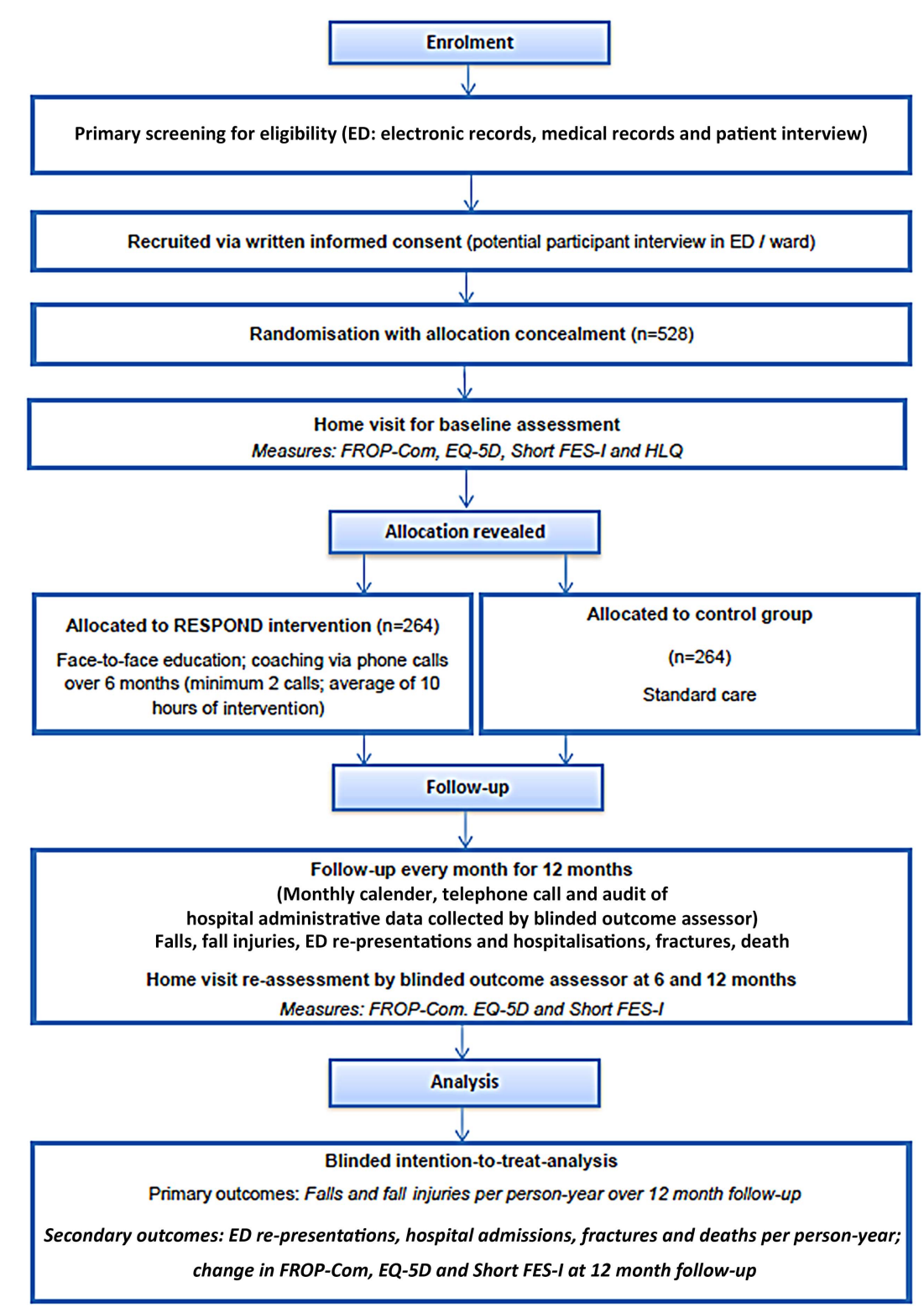

Where FROP-Com = Falls Risk for Older People - Community Setting, EQ-5D = Health-Related Quality of Life Questionnaire, Short FES-I = Shortened Version of Falls Efficacy Scale - International

Functional health literacy will be assessed using the Health Literacy Questionnaire (HLQ), a tool that includes nine conceptually distinct areas of health literacy and has been demonstrated to possess robust psychometric properties. ${ }^{27}$ Health-related quality of life will be assessed using the EQ-5D, a utility-based quality-of-life instrument that estimates quality-adjusted life years and provides a single value for health-related quality of life. ${ }^{28} 29$ Falls self-efficacy will be assessed using the Falls Efficacy ScaleInternational (short version) (Short FES-I). ${ }^{30}$ This seven-item tool measures the level of concern about falling during social and physical activities inside and outside the home and has been shown to be a reliable and valid measure of fear of falling in older people. ${ }^{31}$

The baseline assessment will be conducted in a standard way to minimise the likelihood that it could influence behaviour change in control participants. A simple written report including the participants falls risk status (low, medium or high falls risk) based on the FROP-Com score will be sent to each participant's general practitioner (GP) following baseline assessment. If the participant scored 'moderate or severe anxiety or depression' on the EQ-5D, this information will also be included on the letter. All letters to the GP will be countersigned by a study geriatrician.

\section{Intervention}

The RESPOND programme will be implemented by the RESPOND clinicians over a 6-month period. Table 1 describes the intervention according to the CONSORT extension Template for Intervention Description and Replication guidelines (TIDieR). ${ }^{32}$

The RESPOND clinician will explore participant's falls knowledge, beliefs and self-efficacy to assist in the selection of options for management. The focus will be on participant choice and engagement. Risk factor goals will be based on each participant's individual risk factor profile, social factors, work and/or family commitments and summarised into an individualised action plan. Motivational interviewing will be used to support the participant to understand assessment findings and to facilitate them in 


\begin{tabular}{|c|c|c|c|c|}
\hline \multirow[b]{2}{*}{ Risk Factor } & \multicolumn{4}{|c|}{$\begin{array}{l}\text { Selection of one or more risk factors and management strategies to be addressed by intervention participant } \\
\text { during coaching with RESPOND clinician }\end{array}$} \\
\hline & $\begin{array}{c}\text { 1. Strength and/or balance } \\
\text { impairment }\end{array}$ & 2. Vision impairment & $\begin{array}{l}\text { 3. Long-term use of } \\
\text { Benzodiazepines }\end{array}$ & 4. Poor bone health \\
\hline \multirow[b]{2}{*}{ Risk Assessment } & $\sqrt{3}$ & $\sqrt{n}$ & $\sqrt{n}$ & $\sqrt{n}$ \\
\hline & $\begin{array}{l}\text { Functional mobility } \\
\text { Gait and balance items } \\
\text { from FROP-Com }\end{array}$ & $\begin{array}{l}\text { Vision Screening } \\
\text { Multifocal/Bifocal use in } \\
\text { relation to activity levels }\end{array}$ & Use of Benzodiazepines & $\begin{array}{c}\text { Review of past fall } \\
\text { injuries } \\
\text { Known low trauma } \\
\text { fracture } \\
\text { Serum Vitamin D and/or } \\
\text { DXA and/or FRAX } \\
\text { results }\end{array}$ \\
\hline & $\sqrt{3}$ & $\sqrt{n}$ & $\sqrt{n}$ & 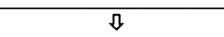 \\
\hline Risk Management & $\begin{array}{l}\text { Exercise program } \\
\text { Gait aid prescription }\end{array}$ & $\begin{array}{l}\text { Vision test and /or review } \\
\text { of current prescription } \\
\text { Ophthalmologist referral } \\
\text { e.g. for cataract surgery } \\
\text { Home safety modifications }\end{array}$ & $\begin{array}{l}\text { Gradual withdrawal or } \\
\text { rationalisation of } \\
\text { Benzodiazepines by GP } \\
\text { 'Sleep Hygiene' Education }\end{array}$ & $\begin{array}{c}\text { Test of Vitamin D levels } \\
\text { +/- Vitamin D } \\
\text { supplementation } \\
\text { Sunlight exposure } \\
\text { Exercise program }\end{array}$ \\
\hline
\end{tabular}

Figure 2 RESPOND risk factor assessment and management foci.

making guided decisions about how they will action recommendations and referrals. Clinicians will also assist in identifying solutions to barriers identified by participants.

The RESPOND clinician will not duplicate care provided by other healthcare professionals involved in the participants care during the 6-month intervention period. The RESPOND clinician assessment will capture existing care recommendations and healthcare professionals involved in the participants care. RESPOND clinicians will refer intervention participants to relevant services and facilitate community linkages.
The participant's ongoing consultation with GPs and specialist physicians over the course of the study will be encouraged. As part of the study intervention, the RESPOND clinician will communicate the individualised action plan to the participant's healthcare providers and any community services the participant is linked into.

\section{The comparator}

Participants in the control group will receive the same baseline assessment as outlined above. A letter detailing the participants

Table 1 Intervention description as per TIDieR ${ }^{32}$

\begin{tabular}{|c|c|}
\hline TIDieR item no & Item \\
\hline 1. Brief name & $\begin{array}{l}\text { RESPOND to the first fall to prevent the second-a patient-centred programme to prevent secondary falls in older people } \\
\text { presenting to the ED with a fall }\end{array}$ \\
\hline 2. Why & $\begin{array}{l}\text { Falls by older people are frequent and associated with disability, institutionalisation and mortality. Older people presenting to } \\
\text { the ED following a fall frequently fall again indicating a failure in secondary falls prevention. This trial will test the efficacy of } \\
\text { delivering patient-centred education and behaviour change strategies to enhance patient engagement in falls prevention } \\
\text { activities. }\end{array}$ \\
\hline 3. What (materials) & $\begin{array}{l}\text { The RESPOND programme targets four risk factors with evidence of effective interventions: poor balance and/or loss of strength; } \\
\text { vision impairment; long-time use of benzodiazepines; and poor bone health. Four education leaflets have been developed } \\
\text { specifically for the project providing simple information on these risk factors and positive health messages relating to } \\
\text { management options. }\end{array}$ \\
\hline 4. What (procedures) & $\begin{array}{l}\text { The RESPOND programme has three components: (1) home-based risk factor assessment; ( } 2 \text { ) education on risk factor } \\
\text { management, goal setting, coaching and follow-up telephone support for management of one or more of four risk factors with } \\
\text { evidence of effective interventions; and ( } 3 \text { ) healthcare provider communication and community linkage into existing community } \\
\text { services that meet participant goals. }\end{array}$ \\
\hline 5. Who provided & $\begin{array}{l}\text { Clinician employed by the RESPOND team. A health professional trained in motivational interviewing and behaviour change } \\
\text { strategies and experienced in falls prevention including completing home safety assessments and prescribing falls prevention } \\
\text { exercises. }\end{array}$ \\
\hline 6. How delivered & $\begin{array}{l}\text { The intervention is personalised and provided on a one-to-one basis; initially face-to-face with subsequent coaching over the } \\
\text { telephone. }\end{array}$ \\
\hline 7. Where delivered & Face-to-face intervention occurs in the participant's home. \\
\hline 8. When and how much & $\begin{array}{l}\text { The clinician will provide an initial } 45 \text { min face-to-face session within } 2 \text { weeks of ED discharge. The first coaching phone call will } \\
\text { be made within } 2 \text { weeks of initial visit and the second within } 3 \text { months. Remaining phone calls will occur at intervals that allow } \\
\text { progress towards goals. There will be a minimum of two follow-up phone calls with each call lasting approximately } 45 \text { min. Each } \\
\text { participant will receive an average of } 10 \text { hours of the intervention. }\end{array}$ \\
\hline 9. Tailoring & $\begin{array}{l}\text { Participants may choose to address one or more of the four risk factors with the option to add in extra strategies throughout the } \\
\text { follow-up period. }\end{array}$ \\
\hline 10. Modifications & Modifications made to the intervention during the course of the study will be reported in the outcome paper. \\
\hline 11. Assessment of intervention fidelity & $\begin{array}{l}\text { A detailed programme evaluation will be conducted concurrently to the RCT to assess if the intervention was implemented as } \\
\text { planned. This evaluation has detailed methodology and will be reported in a separate protocol paper. }\end{array}$ \\
\hline
\end{tabular}


risk status will be provided by the assessing clinician to the control participant's GP following the baseline assessment. Where the participant indicates moderate or severe anxiety or depression on the EQ-5D, this will be communicated in the GP letter. Control participants will receive standard care from all health professionals who are involved in their management within the ED and in the primary care setting during the 12-month follow-up. No treatments will be withheld from the control group. Care in the ED may consist of investigations and multidisciplinary assessment within the ED, referral to other health professionals and services, and postdischarge telephone contact by a nurse. Control participants will not receive any coaching phone calls or other contact from the RESPOND clinician after the baseline assessment.

\section{Outcome measures}

Table 2 outlines the primary and secondary outcomes for this trial, and the timing of their collection. The primary outcomes are falls and fall injuries per person-year in the 12 months after recruitment. A fall will be defined as per the World Health Organisation (WHO), "an event resulting in a person coming to rest inadvertently on the ground, floor or other lower level". ${ }^{33}$ A fall injury is any physical harm resulting from a fall reported by study participants on the monthly calendars or during monthly telephone calls. Where participants suffer multiple injuries from one fall, all injuries will be included in the outcome analysis irrespective of their severity.

Secondary outcomes are ED re-presentations, hospitalisations, fractures (confirmed by radiological investigation) and deaths per-person year in the 12 months post randomisation. Change in falls risk status, falls self-efficacy and health-related quality of life in the 12 months post randomisation will also be evaluated.

\section{Data collection}

Hospital admitted episode and ED administrative data will be audited to obtain the number of potentially eligible study participants, participant demographics and diagnoses, and ED re-presentations and hospitalisations that occur during the follow-up.

Participants in both groups of the trial will complete monthly calendars over the 12-month follow-up documenting details of any falls, fall injuries, ED presentations and hospital admissions on a daily basis. Calendars will be returned monthly by participants using prepaid envelopes. All participants will receive a monthly telephone call to verify information recorded on calendars. This will be conducted by RESPOND outcome assessors who will be blinded to participants' group allocation. Calendar and telephone-verified data on falls, fall injuries, fractures, ED presentations and hospital admissions will be triangulated with data recorded in hospital administrative data sets.

Adverse events spontaneously reported by participants to research staff will be reported to the study steering committee for evaluation.

\section{Statistical analysis}

Outcome analyses will be undertaken on an intention-to-treat basis by a statistician blinded to group allocation. Differences in falls, fall injuries, fractures, ED re-presentation rates and deaths will be compared between groups using negative binomial regression including a variable for adjustment by site. Secondary analysis that adjusts for age and cognitive ability (using FROP-Com cognitive status score obtained at baseline assessment) will be undertaken if significant imbalance in these factors is identified across groups. Differences in continuous outcomes including falls risk, quality of life and falls efficacy scores will be evaluated using General Linear Models (ANCOVA) or the non-parametric Mann-Whitney U statistic where data are not normally distributed. A significance level of $\mathrm{p}<0.05$ will be used for all analyses. The multifactorial design (participants will choose different risk factors and strategies) means it is not possible to discern the effects of any single intervention on the primary outcomes.

Elements introduced to mitigate bias in the study include use of a computer randomisation service and outcome assessment and intention-to-treat analysis performed by staff blinded to participant's group allocation.

\section{Ethics approval}

Ethics approval was obtained from each of the participating hospitals, Alfred Health (HREC 439/13) and Royal Perth Hospital (REG 13-128) and Monash University Human Research Ethics Committee (MUHREC CF13/3869-201300).

Table 2 RESPOND outcome measures and key covariates collected at study time points

\begin{tabular}{|c|c|c|c|c|}
\hline & $\begin{array}{l}\text { Mode of } \\
\text { collection }\end{array}$ & $\begin{array}{l}\text { Collected at } \\
\text { Baseline }\end{array}$ & $\begin{array}{l}\text { Collected during } \\
\text { monthly follow-up }\end{array}$ & $\begin{array}{l}\text { Collected at } \\
6 \text { and } 12 \text { months }\end{array}$ \\
\hline \multicolumn{5}{|l|}{ Primary outcomes } \\
\hline Falls per person-year & $\mathrm{C} ; \mathrm{MT} ; \mathrm{AD}$ & & $\checkmark$ & $\checkmark$ \\
\hline Fall injuries per person-year & $\mathrm{C} ; \mathrm{MT} ; \mathrm{AD}$ & & $\checkmark$ & $\checkmark$ \\
\hline \multicolumn{5}{|l|}{ Secondary Outcomes } \\
\hline $\begin{array}{l}\text { Change in the Falls Risk for Older People in the Community } \\
\text { (FROP-COM) falls risk score }\end{array}$ & $\mathrm{HV}$ & $\checkmark$ & & $\checkmark$ \\
\hline Change in quality of life (EQ-5D) & HV & $\checkmark$ & & $\checkmark$ \\
\hline Change in Falls Efficacy Scale International (shortened FES-I) & HV & $\checkmark$ & & $\checkmark$ \\
\hline Fractures per person-year & $\mathrm{C} ; \mathrm{MT} ; \mathrm{R} ; \mathrm{AD}$ & & $\checkmark$ & $\checkmark$ \\
\hline ED presentations per person-year & C; MT; AD & & $\checkmark$ & $\checkmark$ \\
\hline Hospital admissions per person-year & $\mathrm{C} ; \mathrm{MT} ; \mathrm{AD}$ & & $\checkmark$ & $\checkmark$ \\
\hline Deaths per person-year & $A D$ & & $\checkmark$ & $\checkmark$ \\
\hline \multicolumn{5}{|l|}{ Covariates } \\
\hline Health literacy questionnaire (HLQ) score & HV & $\checkmark$ & & \\
\hline
\end{tabular}




\section{DISCUSSION}

This RCT will develop and test a patient-centred programmeRESPOND—that aims to support older people in making decisions about how they will manage their falls risk. The intervention will assist participants to participate in falls prevention activities by providing education, coaching, referral to services they need and ongoing telephone support to provide positive reinforcement and to troubleshoot barriers that are identified.

Patient-centred models have been successfully trialled in chronic disease $\mathrm{e}^{20}$ and secondary prevention of cardiovascular events. The RESPOND programme draws its conceptual framework from the experience with CHOICE and builds on our previous work addressing patient participation in falls prevention activities. ${ }^{34-39}$ RESPOND will include additional tailoring to the frailer client group who are likely to be the majority of the study sample.

This study design is supported by extensive standard operating procedures (SOPs) developed for the recruiters, clinicians and outcome assessors at each stage of the study. In order to prevent contamination, strategies have been included in the SOPs to ensure that study staff and standard care practitioners do not influence the behaviour of participants in the control group. The main contamination threat to the control group lies in ED staff incorporating some of the intervention strategies into standard care practices. Recruiters have been specifically trained not to flag participants to ED staff and to minimise discussion about potential participants. Randomisation will be concealed from all study staff until after the baseline assessment has been completed and from the outcome assessors and study statistician for the study duration.

A potential source of contamination is provision of information about falls risk to the control participant's GP. While we can argue that this will not change their behaviour, it is not 'usual care' and a failure to show a difference between study groups may be due to individual GPs acting on the information provided about control participants by RESPOND staff.

The study internal validity is strengthened by the inclusion of competency checks for staff adherence to operating procedures. Staff across both study sites will be trained by the same instructor using reference to the SOPs and tools to ensure identical data collection practices. Performance indicators have been developed for each of the study roles (ie, recruiter, clinician, outcome assessor) and compliance with SOPs will be verified by quality audits at each stage of the study.

Since recall bias has the potential to limit accuracy of data, this study has applied current best practice recommendations for identifying fall events, which involves the use of multiple methods for the capture of falls data. ${ }^{40}$ Participants will record fall events prospectively in a study calendar, rather than relying on recollection at follow-up time points, and this information will be verified by outcome assessors during the monthly phone calls. Fall injuries that result in an ED hospital presentation will be triangulated with hospital administrative data. Participants with cognitive impairment have been excluded to minimise bias associated with memory impairment.

Our findings will generate new knowledge on strategies to enhance care of older people who present to the ED after a fall and who are likely to fall again. However, findings may not be generalisable to all community-dwelling older people who fall, or to frail older people who are in residential aged care.

The project will also investigate the cost-effectiveness, acceptability and sustainability of the RESPOND programme, as well as participant knowledge, attitudes and beliefs surrounding participation in falls prevention activities. These investigations have detailed methodology in addition to that reported here and will be described in subsequent protocols.

The research outcomes have the potential to change current falls prevention practice and policies for older people presenting to an ED with a fall. The findings from this project could impact on the planning, design, implementation and management of secondary falls prevention programmes in Australia and internationally.

\section{Author affiliations}

${ }^{1}$ Health Services Research Unit, Centre of Research Excellence in Patient Safety, Division of Health Services and Global Health Research, Department of Epidemiology and Preventive Medicine, Monash University, Melbourne, Victoria, Australia ${ }^{2} S$ chool of Physiotherapy and Exercise Science, Curtin University of Technology, Perth, Western Australia, Australia

${ }^{3}$ University of Western Australia, Perth, Western Australia, Australia

${ }^{4}$ Geriatric Medicine, Royal Perth Hospital, Perth, Western Australia, Australia ${ }^{5}$ Harry Perkins Institute for Medical Research, Perth, Western Australia, Australia ${ }^{6}$ Department of Physiotherapy, Monash University, and Allied Health Research Unit, Monash Health, Melbourne, Victoria, Australia

${ }^{7}$ Health Networks Branch, System Policy and Planning, Department of Health, Government of Western Australia, Perth, Western Australia, Australia

${ }^{8}$ The George Institute for Global Health, Sydney Medical School, University of Sydney, Camperdown, New South Wales, Australia

${ }^{9}$ Department of Epidemiology and Preventive Medicine, Monash University, Melbourne, Victoria, Australia

${ }^{10}$ Melbourne EpiCentre, University of Melbourne and Melbourne Health, Melbourne, Victoria, Australia

${ }^{11}$ School of Physiotherapy, The University of Notre Dame Australia, Fremantle, Western Australia, Australia

${ }^{12}$ Alfred Health, Melbourne, Victoria, Australia

${ }^{13}$ Bournemouth University Dementia Institute and Psychology Department, Faculty of Science and Technology, Bournemouth University, Poole, Dorset, UK

Acknowledgements We thank Dr Gayle Savige for assistance in preparing this manuscript for publication and the representatives from our funding partner organisations who have contributed to development and implementation of this study. The partner organisations include

1. Health Strategy and Networks Branch, Strategic System Policy and Planning, Department of Health, WA

2. Aged and Continuing Care Directorate, Department of Health, WA

3. Royal Perth Hospital

4. Curtin University of Technology

5. The University of Western Australia

6. The Royal Perth Hospital Medical Research Foundation

7. Sir Charles Gairdner Hospital (SCGH) Area Rehabilitation and Aged Care Falls Specialist Program

8. Injury Control Council of Western Australia (ICCWA)

9. The George Institute for Global Health

10. The Alfred Hospital

11. Monash University

12. Integrated Care, Victorian Department of Health

Contributors All authors provided substantial contribution to conception and design of the project, drafting the article and revising it critically for important intellectual content; and final approval of the version to be published. $A B$ led the drafting of all sections of the article in consultation with all of the co-authors. $\mathrm{KH}$, JR and SN provided substantial contributions to the background, critical appraisal of prior studies and rationale for the project. PC, GA, JL, PH, DS and AF provided substantial contribution to the design of the recruitment processes. CB, NW, SN, LF, $\mathrm{JR}, \mathrm{A}-\mathrm{MH}$ and CEB provided substantial contribution to the intervention design. $\mathrm{KH}, \mathrm{CB}, T H, A F$, JR and JL provided substantial contribution to the overall design aspects including selection of outcome measures and refinement of study processes. $A B$ and $A F$ provided substantial contribution to the sample size and statistical analysis section.

Funding This project is funded under the Australian National Health and Medical Research Council (NHMRC) Partnership Projects funding scheme (project number APP1056802) with financial and in-kind contributions from the partner organisations listed above. AB is funded by a Career Development Fellowship funded by the NHMRC (1067236), JR is funded by a Postdoctoral Fellowship co-funded by the NHMRC and National Heart Foundation (632933) and JAL is funded by an Early Career Fellowship funded by the NHMRC (1052442).

Competing interests None. 
Ethics approval The Alfred Hospital Ethics Committee; Royal Perth Hospital Human Research Ethics Committee; Monash University Human Research Ethics Committee.

Provenance and peer review Not commissioned; externally peer reviewed.

\section{REFERENCES}

1 Samaras N, Chevalley T, Samaras D, et al. Older patients in the emergency department: a review. Ann Emerg Med 2010;56:261-9.

2 Bloch $F$, Jegou D, Dhainaut JF, et al. Do ED staffs have a role to play in the prevention of repeat falls in elderly patients? Am J Emerg Med 2009;27:303-7.

3 Close J, Ellis M, Hooper R, et al. Prevention of falls in the elderly trial (PROFET): a randomised controlled trial. Lancet 1999;353:93-7.

4 Russell MA, Hill KD, Day LM, et al. A randomized controlled trial of a multifactorial falls prevention intervention for older fallers presenting to emergency departments. J Am Geriatr Soc 2010;58:2265-74.

5 Russell MA, Hill KD, Day LM, et al. Development of the Falls Risk for Older People in the Community (FROP-Com) screening tool. Age Ageing 2009;38:40-6.

6 Hendriks MR, Bleijlevens MH, van Haastregt JC, et al. Lack of effectiveness of a multidisciplinary fall-prevention program in elderly people at risk: a randomized, controlled trial. J Am Geriatr Soc 2008;56:1390-7.

7 Shaw FE, Bond J, Richardson DA, et al. Multifactorial intervention after a fall in older people with cognitive impairment and dementia presenting to the accident and emergency department: randomised controlled trial. BMJ 2003;326:73.

8 Whitehead C, Wundke R, Crotty M, et al. Evidence-based clinical practice in falls prevention: a randomised controlled trial of a falls prevention service. Aust Health Rev 2003;26:88-97.

9 de Vries OJ, Peeters GM, Elders PJ, et al. Multifactorial intervention to reduce falls in older people at high risk of recurrent falls: a randomized controlled trial. Arch Intern Med 2010;170:1110-17.

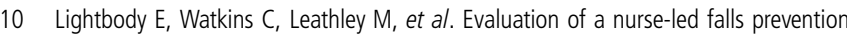
programme versus usual care: a randomized controlled trial. Age Ageing 2002:31:203-10.

11 Pardessus V, Puisieux F, Di Pompeo C, et al. Benefits of home visits for falls and autonomy in the elderly: a randomized trial study. Am J Phys Med Rehabil 2002;81:247-52

12 Davison J, Bond J, Dawson P, et al. Patients with recurrent falls attending Accident \& Emergency benefit from multifactorial intervention — a randomised controlled trial Age Ageing 2005;34:162-8.

13 Prince RL, Austin N, Devine A, et al. Effects of ergocalciferol added to calcium on the risk of falls in elderly high-risk women. Arch Intern Med 2008;168:103-8.

14 Gates S, Fisher JD, Cooke MW, et al. Multifactorial assessment and targeted intervention for preventing falls and injuries among older people in community and emergency care settings: systematic review and meta-analysis. BMJ 2008;336:130-3.

15 Vind $A B$, Andersen $H E$, Pedersen $K D$, et al. An outpatient multifactorial falls prevention intervention does not reduce falls in high-risk elderly Danes. J Am Geriatr Soc 2009;57:971-7.

16 Whitehead $\mathrm{CH}$, Wundke R, Crotty M. Attitudes to falls and injury prevention: what are the barriers to implementing falls prevention strategies? Clin Rehabil 2006;20:536-42

17 Hill K. Don't lost sight of the importance of the individual in effective falls prevention interventions. BMC Geriatrics 2009;9:13.

18 Mead N, Bower P. Patient-centredness: a conceptual framework and review of the empirical literature. Soc Sci Med 2000;51:1087-110.

19 Bunn F, Dickinson A, Barnett-Page E, et al. A systematic review of older people's perceptions of facilitators and barriers to participation in falls-prevention interventions. Ageing Soc 2008;28:449-72.

20 Barlow JH, Wright CC, Turner AP, et al. A 12-month follow-up study of self-management training for people with chronic disease: are changes maintained over time? Br J Health Psychol 2005;10(Pt 4):589-99.
21 Redfern J, Briffa T, Ellis E, et al. Choice of secondary prevention improves risk factors after acute coronary syndrome: 1-year follow-up of the CHOICE (Choice of Health Options In prevention of Cardiovascular Events) randomised controlled trial. Heart 2009;95:468-75.

22 Neubeck L, Freedman SB, Briffa T, et al. Four-year follow-up of the Choice of Health Options In prevention of Cardiovascular Events randomized controlled trial. Eur J Cardiovasc Prev Rehabil 2011;18:278-86.

23 Simek EM, McPhate L, Haines TP. Adherence to and efficacy of home exercise programs to prevent falls: a systematic review and meta-analysis of the impact of exercise program characteristics. Prev Med 2012;55:262-75.

24 Tombaugh TN, Mclntyre NJ. The mini-mental state examination: a comprehensive review. J Am Geriatr Soc 1992;40:922-35.

25 Molloy DW, Clarnette R. Standardized Mini-Mental State Examination: a user's guide. Troy, NY: New Grange Press; 1999.

26 Russell MA, Hill KD, Blackberry I, et al. The reliability and predictive accuracy of the falls risk for older people in the community assessment (FROP-Com) tool. Age Ageing 2008;37:634-9.

27 Osborne RH, Batterham RW, Elsworth GR, et al. The grounded psychometric development and initial validation of the Health Literacy Questionnaire (HLQ). BMC Public Health 2013;13:658.

28 Hurst NP, Kind P, Ruta D, et al. Measuring health-related quality of life in rheumatoid arthritis: validity, responsiveness and reliability of EuroQol (EQ-5D). Br J Rheumatol 1997;36:551-9.

29 Tidermark J, Bergstrom G. Responsiveness of the EuroQol (EQ-5D) and the Nottingham Health Profile (NHP) in elderly patients with femoral neck fractures. Qual Life Res 2007;16:321-30.

30 Kempen GI, Yardley L, van Haastregt JC, et al. The Short FES-I: a shortened version of the falls efficacy scale-international to assess fear of falling. Age Ageing 2008;37:45-50

31 Helbostad JL, Taraldsen K, Granbo R, et al. Validation of the Falls Efficacy Scale-International in fall-prone older persons. Age Ageing 2010;39:259.

32 Hoffmann TC, Glasziou PP, Boutron I, et al. Better reporting of interventions: template for intervention description and replication (TIDieR) checklist and guide. BMJ 2014;348:g1687.

33 World Health Organization. WHO Global Report on Falls Prevention in Older Age. Secondary WHO Global Report on Falls Prevention in Older Age 2007, page 1. http://www.who.int/violence_injury_prevention/other_injury/falls/en/index.html

34 Hill AM, Hoffmann T, McPhail $\bar{S}$, et al. Factors associated with older patients' engagement in exercise after hospital discharge. Arch Phys Med Rehabil 2011;92:1395-403.

35 Hill AM, Hill K, Brauer $S$, et al. Evaluation of the effect of patient education on rates of falls in older hospital patients: description of a randomised controlled trial. BMC Geriatr 2009;9:14.

36 Hill AM, McPhail S, Hoffmann T, et al. A randomized trial comparing digital video disc with written delivery of falls prevention education for older patients in hospital. J Am Geriatr Soc 2009;57:1458-63.

37 Haines TP, Hill AM, Hill KD, et al. Patient education to prevent falls among older hospital inpatients: a randomized controlled trial. Arch Intern Med 2011;171:516-24.

38 Nyman SR, Victor CR. Older people's recruitment, sustained participation, and adherence to falls prevention interventions in institutional settings: a supplement to the Cochrane systematic review. Age Ageing 2011;40:430-6.

39 Nyman SR, Victor CR. Older people's participation in and engagement with falls prevention interventions in community settings: an augment to the Cochrane systematic review. Age Ageing 2012;41:16-23.

40 Lamb SE, Jørstad-Stein EC, Hauer K, et al. Prevention of Falls Network Europe and Outcomes Consensus Group. Development of a common outcome data set for fall injury prevention trials: the Prevention of Falls Network Europe consensus. J Am Geriatr Soc 2005;53:1618-22. 